but differ for insignificant cancer. In the study from Korea, the frequency of organ-confined disease was $97 \%$ (vs. 92\% from Johns Hopkins) but 69\% for insignificant cancer (vs. 84\% from Johns Hopkins).

\title{
References
}

1. Schröder FH, Hugosson J, Roobol MJ, Tammela TL, Ciatto S, Nelen V, et al.: Screening and prostate-cancer mortality in a randomized European study. N Engl J Med. 2009; 360: 1320-8.

2. Bastian PJ, Mangold LA, Epstein JI, Partin AW: Characteristics of insignificant clinical T1c prostate tumors. A contemporary analysis. Cancer. 2004; 101: 2001-5.

Dr. Athanase Billis

Full-Professor of Pathology

State University of Campinas, Unicamp

Campinas, São Paulo, Brazil

E-mail: athanase@fcm.unicamp.br

doi: 10.1590/S1677-55382010000700022

\section{The Epstein criteria predict for organ-confined but not insignificant disease and a high likelihood of cure at radical prostatectomy}

Lee MC, Dong F, Stephenson AJ, Jones JS, Magi-Galluzzi C, Klein EA

Glickman Urological and Kidney Institute, Cleveland Clinic, Cleveland, OH, USA

Eur Urol. 2010; 58: 90-5

Background: Few reports attempt to validate the role of Epstein criteria in selecting patients for an active surveillance protocol.

Objective: To determine the performance of the Epstein biopsy criteria for predicting pathologic end points and biochemical relapse-free survival (bRFS) in men with early stage prostate cancer (PCa) treated by radical prostatectomy (RP).

Design, Setting and Participants: Between October 1999 and January 2007, 746 consecutive patients were biopsied, and then underwent RP at our tertiary care institution. Two hundred sixty-eight patients met the entry criteria of Gleason 6 disease only on initial biopsy with complete pathologic information.

Measurements: Primary end point was insignificant disease. Insignificant disease was defined using a classical (organ-confined, Gleason score $<6$, and tumor volume $<0.5 \mathrm{~cm}(3)$ ) and more liberal (organ-confined, Gleason $<6$ tumor of any volume) formulation. Secondary end points included organ-confined disease and bRFS.

Results and Limitations: One hundred thirty-six men (51\%) met the Epstein biopsy criteria, and 167 (62\%) had organ-confined cancer. Insignificant disease by the classical and liberal definitions was present in $68(25 \%)$ and 92 (34\%) patients, respectively. Cases meeting Epstein biopsy criteria were more likely to have insignificant disease by either definition $(\mathrm{p}<0.001)$ and more likely to have organ-confined tumors $(\mathrm{p}<0.001)$. Sensitivity, specificity, positive predictive value (PPV), and negative predictive value (NPV) varied widely among the end points, with sensitivity (74\%) and NPV (86\%) best for the classical definition of insignificant disease and specificity (74\%) and PPV (92\%) best for organ-confined disease. The estimated 5-yr bRFS was 100\% for those meeting Epstein biopsy criteria compared to $83 \%$ for those not meeting these criteria.

Conclusions: The Epstein biopsy criteria predict for a high likelihood of organ-confined disease and the absence of biochemical failure up to 5 years after RP. These criteria are insufficiently robust to predict the presence of biologically insignificant disease. 


\title{
Editorial Comment
}

The conclusion of Lee's et al. study from Cleveland Clinic is that Epstein biopsy criteria predict for a high likelihood of organ-confined disease but are insufficiently robust to predict the presence of insignificant disease defined as organ-confined, Gleason low-grade, and minimal volume $(\leq 0.5 \mathrm{cc})$.

The findings are supported by other studies (1-3). Epstein's criteria are highly predictive for organ-confined prostate cancer. The frequency varies from $91 \%$ to $97 \%$. However, the predictive value for insignificant cancer varies from $37 \%$ to $84 \%$. Jeldre's et al. concluded that Epstein's criteria might underestimate the true nature of prostate cancer in as many as $24 \%$ of European patients (1). Approximately $31 \%$ Korean patients who meet all the conditions of the contemporary Epstein's criteria for prediction of clinically insignificant prostate cancer may actually harbor prostate cancer with unfavorable pathological features (2). In the Middle East (Egypt), $46 \%$ of patients may present unfavorable cancer (3).

There are several causes for the discrepancies. Prostate cancers diagnosed in Asian, American, and European men may have innate differences associated with racial and/or environmental factors. However, methodological factors seem to be more important: among others, number of patients studied, number of cores of the biopsy, and criteria for volume evaluation. The last one was considered by Lee's et al. study from the Cleveland Clinic.

According to volume, the authors defined prostate cancer by two ways: classical and liberal. The classical definition considered a tumor volume $<0.5 \mathrm{cc}$; and the liberal definition any grade of volume. Using the liberal definition, the predictive value of Epstein's criteria for insignificant cancer was 58\%; using the classical definition was $37 \%$. In a similar study at our Institution (data not published), the frequency was $55 \%$ and $46 \%$, respectively.

\section{References}

1. Jeldres C, Suardi N, Walz J, Hutterer GC, Ahyai S, Lattouf JB, et al.: Validation of the contemporary epstein criteria for insignificant prostate cancer in European men. Eur Urol. 2008; 54: 1306-13.

2. Lee SE, Kim DS, Lee WK, Park HZ, Lee CJ, Doo SH, et al.: Application of the Epstein criteria for prediction of clinically insignificant prostate cancer in Korean men. BJU Int. 2010; 105: 1526-30.

3. Hekal IA, El-Tabey NA, Nabeeh MA, El-Assmy A, Abd El-Hameed M, Nabeeh A, et al.: Validation of Epstein criteria of insignificant prostate cancer in Middle East patients. Int Urol Nephrol. 2010; 42: 667-71.

\author{
Dr. Athanase Billis \\ Full-Professor of Pathology \\ State University of Campinas, Unicamp \\ Campinas, São Paulo, Brazil \\ E-mail: athanase@fcm.unicamp.br
}

RECONSTRUCTIVE UROLOGY

doi: $10.1590 / S 1677-55382010000700023$

The use of penile skin graft versus penile skin flap in the repair of long bulbo-penile urethral stricture: a prospective randomized study

Hussein MM, Moursy E, Gamal W, Zaki M, Rashed A, Abozaid A

Urology Department, Sohag Faculty of Medicine, Sohag University, Sohag, Egypt

Urology. 2011 Jan 3. [Epub ahead of print] 